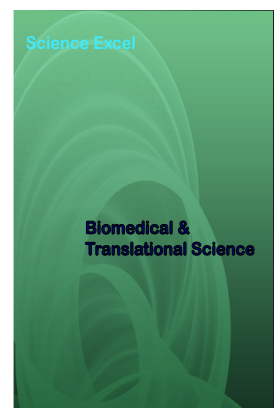

Correspondence

Marjan Assefi

University of North Carolina, Greensboro, North Carolina, USA

E-mail: massefi@aggies.ncat.edu

\title{
Potential Clinical Usefulness of CRISPR/Cas9 Genome Editing in Cancer Treatment
}

\author{
Marjan Assefi ${ }^{12^{*}}$, Sahar Asvadi', Hossein Ghahramani Almanghadim ${ }^{1}$, Fatemeh \\ Zeinali Sehrig ${ }^{1}$, Zahra Foruzandeh ${ }^{1}$, Shahab Masoumi ${ }^{3}$, Hadis Sheikhi'
}

'Department of Medical Genetics, Faculty of Medicine, Tabriz University of Medical Sciences, Tabriz, Iran, 'University of North Carolina at Greensboro, Greensboro, North Carolina, USA

${ }^{3}$ University of California, Berkeley, Berkeley, USA

\begin{abstract}
Cancer is still considered a main challenge regarding morbidity and mortality, although an earlier diagnosis through screening programs and more effective cure modalities have led to decreased mortality rates so the development of new genetic editing techniques such as zinc finger and TALENs or CRISPRCas9 has enabled the production of strong animal genetic models that sums up the cooperating oncogenic lesions influencing genes with a confirmed role in the proliferation and formation. This review presents the mechanisms of separate genome-editing approaches and explains each of the common nucleasebased platforms.
\end{abstract}

\section{Introduction}

Cancer has become one of the major causes of global mortality and morbidity. In 2012, 14.1 million new diagnoses and 8 million worldwide deaths could be related to cancer [1]. Cancer is still considered a main challenge regarding morbidity and mortality, although an earlier diagnosis through screening programs and more effective cure modalities have led to decreased mortality rates [1]. More than 600 cancer-inducing somatic mutations which have been listed online has been recognized in a joint global attempt.

The most significant treatment modalities are surgery, radio- and chemotherapy and in in previous years also immunotherapy [2].

Cre/LoxP techniques, zinc finger nucleases (ZFNs) and transcription activator-like effector nucleases (TALENs) are conventional genome editing tools which have been utilized for cancer modelling, and in some cases for therapy explorations [3].

CRISPRCas9 (clustered regularly interspaced short palindromic repeatsCRISPR associated nuclease 9) is an RNAguided genome editing tool which has been recently added to the toolbox [3].

Three types (I-III) of CRISPR systems were first found in bacteria and archaea where they acted as an immune defense system conferring resistance to foreign viruses (e.g., phages) and other genetic elements. It's easy design, high efficiency targeting, and low off-target mutation frequency have promptly made the CRISPR/Cas9 system a most commonly approach suitable for gene-editing. Therefore, the CRISPR/Cas9 has been widely used as a genetic engineering tool in studies of human diseases [4].

The development of new genetic editing techniques such as TALENs or CRISPR-Cas9 has enabled the production of strong animal genetic models that sums up the cooperating oncogenic lesions influencing genes with a confirmed role in the proliferation and formation of the leukemic clone [5].

But, genome editing techniques have further developed and have been used with therapeutic and clinical methods. Their application has made the design of new therapies such as chimeric antigen receptors (CARs) easier and have made the study of genes involved in pathogenesis possible [5].

This review presents the mechanisms of separate genome-editing approaches and explains each of the common nuclease-based platforms, containing zinc finger nucleases, transcription activator-like effector nucleases (TALENs), meganucleases, and the CRISPR/ Cas9 system.

\section{Functional DNA editing in cell}

\section{Zinc finger nucleases}

The first ZNF was recognized in the late 1980s. The first identified ZNF was Transcription Factor IIIa (TFIIIa) from Xenopuslaevis. This led to the discovery of 
a new group of transcriptional activator proteins with a 30 amino acid repeating region [6].

Zinc finger $(\mathrm{ZF})$ proteins are the most plentiful class of transcription factors and the Cys2 -His2 zinc finger domain is one of the most prevalent DNA-binding domains encoded in the human genome [7].

There are about 30 amino acids in a configuration in each zinc-finger motif, and a single zinc atom in individual zincfinger is coordinated by two cysteines and two histidines.

Amino acids in positions $-1,3$, and 6 on the -helix contact 3 base pairs in the main groove of DNA. Therefore, theoretically, a combination of 6 different zinc-fingers that each identifies a 3 base pair DNA sequence can consequently identify a particular DNA sequence of 18 base pairs, which is a long enough sequence to identify a distinctive site in the human genome [8].

Zinc finger nuclease consists of two domains: DNA binding domain with continual zinc fingers and FokI restriction enzyme-derived nuclease domain which is regarded one of the most abundant DNA binding motifs in eukaryotic genome having the ability to identify any sequence [9].

When the cleavage domain of Fok I endonuclease was joined with zinc-finger protein to produce zinc-finger-Fok I fusion proteins, these hybrid fusion proteins (ZFNs) were able to cut DNA at the pre-established sequences [8].

In recent times, three large sets of constructed ZFNproteins (ZFPs) from independent groups, namely The Scripps Research Institute, Sangamo Biosciences, and ToolGen, are available [8].

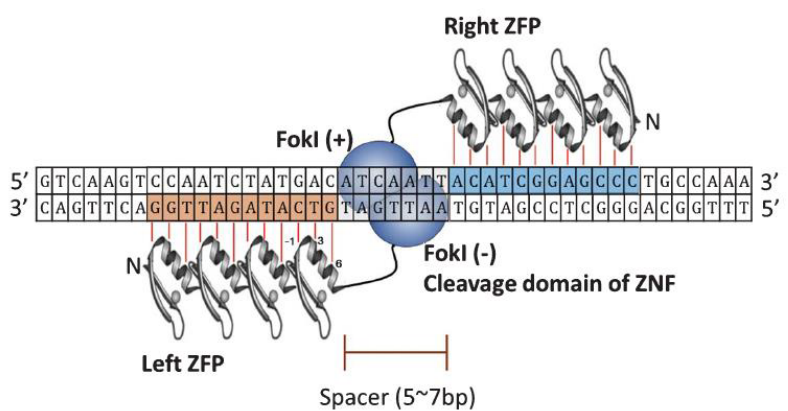

Transcription activator-like effector nucleases (TALENs)

DAnother common SSN for genetic targeting is Transcription activator-like effector (TALE) nucleases (TALENs) which is another common SSN for genetic targeting and comprises both DNA-binding domain and nuclease domain [9].

Fundamentally, TALENs encompasses a domain that activates the target gene transcription (transcription activatorlike effector [TALE]), a DCD, and a nuclear localization signal TALENs produce DSB at specific loci, which begins the DNA repair machinery, by that these mutations are transmitted via the germ line[7]

In comparison to ZFNs, TALENs have only four kinds of RVDs to cover the four nucleotides. This superiority makes it much easier to produce TALEN-targeting clones for gene targeting. A lot of strategies have been developed for TALEN assembly to control problems of assembling continuously repeating residues, including the Golden Gate method, Platinum Gate method, and ligation-independent cloning [7] [10].
The DNA-binding domain of TALE proteins, derived from the plant bacterium, Xanthomonas, incorporate a series of repeating residues with $33^{\star} 35$ amino acids. The 12 th and 13th amino acids within a TALE residue are inconstant and play main roles in DNA recognition, called repeat variable di-residues (RVDs). Each RVD can particularly identify a nucleic acid Asn-Gly for thymine, His-Asp for cytosine, AsnIle for adenine, and Asn-Asn for guanine. Combinations of continuous designed TALE theoretically can identify all DNA sequences. After DNA-binding, DNAs with DSB nicks can be broken and HDR or NHEJ DNA repair reactions41 can be induced by the FokI nuclease[11].

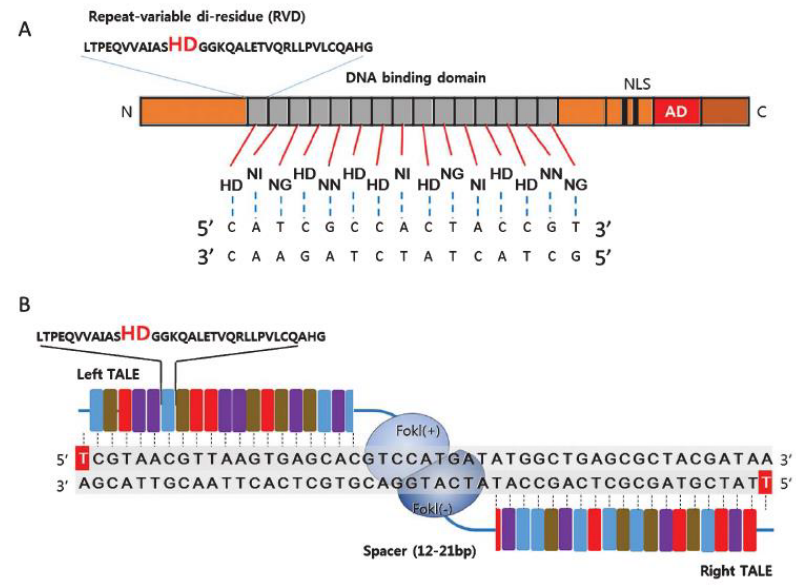

CRISPR/Cas system operates as adaptive immune system in various bacteria and archaea, of which RNAs harboring "spacer" sequence from previously exposed bacteriophages assist Cas proteins identify and cleave the particular exogenous DNA [8]

CRISPR-Cas systems are divided into two classes. While types I, III and IV are included in the class 1 system, types II, V, and VI are involved in the class 2 system. Moreover, the class 1 CRISPR-Cas system utilizes a complex of several Cas proteins, whereas the class 2 system merely makes use of a single Cas protein with multiple domains.

Thus, the class 2 CRISPR-Cas system is better for geneengineering applications due to its simplicity and ease of use.

The type II CRISPR-Cas9 is the most widely studied and utilized system among different kinds of the class 2 CRISPRCas systems. In this system, CRISPR spacers control the system to the target, and the Cas9 protein controls spacer acquisition and defense. Natural CRISPR systems function in three stages, involving adaptation, expression and interference [12].

CRISPR/Cas9, a type II CRISPR/Cas system, initially uses CRISPR RNA (crRNA) and trans-activating crRNA (tracrRNA) to generate crRNA-tracrRNA duplex and then helps Cas9 nuclease to distinguish and cleave target DNA harboring trinucleotideprotospacer adjacent motif (PAM) and a 5' end of 20 nucleotides complementary to the spacers. System reprogramming that fuses crRNAandtracrRNA into a synthetic single guide RNA (sgRNA) considerably facilitates production of transcripts and remarkably promotes the application of CRISPR/Cas9 system .Reprogrammed CRISPR/ Cas9 system has since beensuccessfully utilized in numerous 
organisms, including S. cerevisiae, Drosophila melanogaster, Caenorhabditiselegans, plants ,and human embryos [13].

Using a plasmid-based CRISPR-Cas9 system encoding the Cas9 protein and sgRNA from the same vector is the first and the most straightforward approach which is able to avoid multiple transfections of different components.

There are three strategies to edit genome utilizing CRISPRCas9. The first and the best straightforward method is using a plasmid-based CRISPR-Cas9 system encoding the Cas9 protein and sgRNA from the same vector, therefore avoiding several transfections of different components .The second strategy is to deliver the mixture of the Cas9 mRNA and the sgRNA.The third strategy is to deliver the mixture of the Cas9 protein and the sgRNA [12]

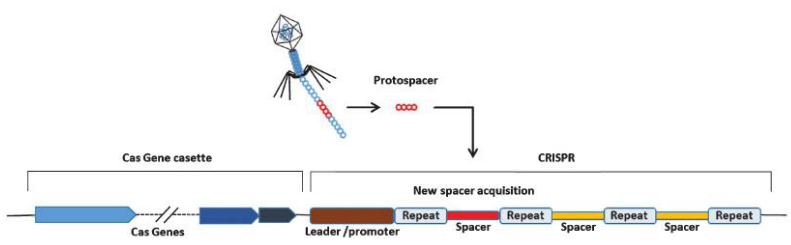

The role of DNA editing in diagnosis and treatment of cancer

Almost all patients with oral cancer only become aware of their condition when the disease has relatively developed or at least at an advanced stage. Surgical and chemo-therapy/ radiotherapy are the most common therapies. However, these therapies are accompanied by severe side effects. Thus, the identification of target based therapies and earlier detections of oral cancer disease would seem to be important for the prevention or at least curing the disease at its very onset [14].

Currently, while the principles have caused the development of the molecularly targeted drugs for clinical practice, the main challenge is the recognition of the anticancer biomarkers as targets for a precise treatment; therefore, it is possible to minimize the overall cost and the side effects associated with the off-target treatments.

It is fortunate that the development of a genome editing technology with the clustered regularly interspaced short palindromic repeats (CRISPR) which are the associated protein nuclease (Cas9) or CRISPR/Cas9 system has made the identification easier [15].

Genomic diversity of cancer controls its addictions to main cellular events. These events involve transcription, splicing, translation, protein folding/degradation, and cell proliferation.

The busy cancer proliferation and tumorigenesis are not merely fueled by the drivers and passengers but they are encoded by the complex of the mutated genes and influenced by other noncoding factors in cancer cells as well. This imposes "rush hours" on these molecular processes and renders the cancer cells addicted to them. These addictions can be interrupted by CRISPR-mediated genome editing (green arrows) [15].

Tumor-specific gene expression programs validated with the CRSPR/Cas genome editing technology have been identified in numerous tumors involving breast basal-like, serous ovarian cancers and lung squamous cell carcinoma [15]

Since the drivers of tumorigenesis and the tumor

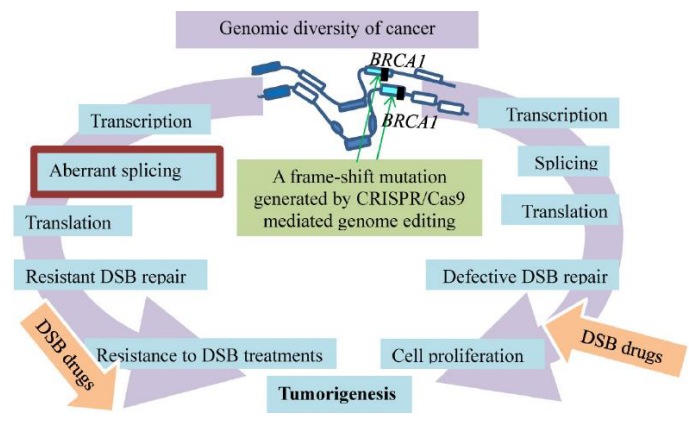

microenvironment are complex and persistently changing, designing an effective cancer therapy is challenging [16].

Challenges to CRISPR-Based Therapeutics

To design an efficacious therapy with the potential for its clinical translation, the treatment requires to be effective against the disease of interest and deliverable to the target of interest, with endurable or no significant adverse effects.

So far, CRISPR/Cas9-based therapies have been associated with certain main restrictions, such as the targeting specificity and the possibility of off-target binding of Cas9 protein, ontarget editing efficiency, and the induction of the host immune response against the delivery vectors [16].

Diagnostic applications

Mutant DNA is considered as a biomarker in cancer and prenataldiagnosis at the present time.

The detection of mutant DNA is serious for precision medicine. However, the determination of low-frequency DNA mutation is very difficult.

The problem of low-frequency mutation detection becomes growingly critical with the evolution of non-invasive diagnosis and individualized medicine.

The detection of low-abundance mutation is frequently restricted by the high background of wild-type DNA in some special clinical samples such as plasma.

Almost all mutations related to diseases are deletion mutation and SNP. For example, in NSCLC patients $46 \%$ of the EGFR mutations are deletion mutation .The golden standard" of genotyping is direct sequencing, which is not enough for low-frequency mutation. A lot of methods have been applied in the last decades in order to overcome this problem such as PNA/LNA-mediated real-time PCR clamping, allele specific PCR and RFLP analysis.

With the progress of CRISPR/Cas9 system, CRISPR/Cas9 has considerable potential in the area of gene detection [17].

Therefore, CRISPR/Cas9 cleavage in vitro might be the best possible choice for mutation enrichment and detection due to its high sensitivity and convenience.

Like traditional T7 endonuclease I (T7E1) assay in appraising mutant DNA proportion in the condition of normal frequency, the system of CRISPR/Cas9 cleavage in vitro revealed a high accuracy. The technology was additionally utilized for lowfrequency mutant DNA detection of EGFR and HBB somatic mutations.

To this end, Cas9 was used to cleave the wild-type (WT) DNA and to enrich the mutant DNA. The sensitivity of 
CRISPR/Cas9 cleavage-based PCR was evaluated using amplified fragment length polymorphism analysis (AFLPA) and Sanger sequencing, in which mutations at 10\%-1\% could be enriched and detected. Its sensitivity reached up to $0.1 \%$ when combined with blocker PCR. The results indicated that this new application of CRISPR/Cas9 system is a vigorous and potential method for heterogeneous specimens in the clinical diagnosis and treatment management.

The two applications of CRISPR/Cas9 cleavage in vitro demonstrated that it is an innovative and exact tool in gene detection [17]

\section{Therapeutic applications}

IThe CRISPR-Cas9 system has quickly attracted the most attention from scientists in different fields and started a race to harness CRISPR-Cas9 for therapeutic applications in humans due to its simplicity, ease of use and potent gene-editing capability.

Basically, the CRISPR-Cas9 system holds high promise for human gene therapy. For instance, it has been effectively employed to correct gene mutations that drive the development of cancers. Also, it has been used to produce oncolytic viruses to transduce and kill tumor cells selectively. The first clinical study of CRISPR-Cas9 was initiated by injecting Cas9engineered $\mathrm{T}$ cells to a patient with metastatic non-small cell lung cancer (NSCLC) by a Chinese group in 2016. As the result was promising A second injection was arranged.

Different strategies to edit genes using the CRISPR-Cas9 system. The first and the most straightforward approach is to use a plasmid-based CRISPR-Cas9 system encoding the Cas 9 protein and sgRNA from the same vector, thus avoiding multiple transfections of different components. The vector will express the Cas9 protein and sgRNA, which will form the Cas9/ sgRNA complex inside cells to edit genomic sequences. The second strategy is to deliver the mixture of the Cas9 mRNA and sgRNA. The Cas9 mRNA will be translated to Cas 9 protein in cells to from the Cas9/sgRNA complex. The third strategy is to directly deliver the Cas9/sgRNA complex into cells [17].

The utilization of a plasmid-based CRISPR-Cas9 system encoding the Cas9 protein and sgRNA from the same vector and consequently avoiding multiple transfections of different components is the first and most straightforward approach. [18]. The second approach is to provide the mixture of the Cas9 mRNA and the sgRNA [19] and the last strategy is delivering the mixture of the Cas9 protein and the sgRNA [20].

In the pioneering stage of the CRISPR-Cas9 system applications, several physical and non-viral delivery approaches such as electroporationm [21] nanoparticles and hydrodynamic injection [22] have been utilized for delivering CRISPR-Cas9 to target cells. The typically used physical and non-viral delivery approaches are discussed below.

\section{Electroporation}

Electroporation is appropriate for all kinds of CRISPRCas9 systems, including plasmid-based CRISPR-Cas9 systems, the mixture of Cas 9 mRNA and sgRNA, and the Cas9/sgRNA RNPs. The limitation of this delivery approach is that plasmid DNA is merely integrated into about $0.01 \%$ of the target cells. Additionally, electroporation causes significant cell death.
Electroporation of RNPs into specific target cells reaches a higher gene-editing effectiveness than electroporation of the corresponding plasmid-based CRISPRR-Cas9 or Cas9 mRNA/ sgRNA. For example, electroporation of RNPs reached editing efficiencies of $87 \%$ and $94 \%$ in produced pluripotent stem cells and Jurkat T cells, respectively. In contrast to electroporation of RNPs electroporation of the plasmid-based CRISPRCas9 system and the Cas9 mRNA/sgRNA induced a lower effectiveness in produced pluripotent stem cells (20\% and $32 \%$, respectively) and Jurkat $\mathrm{T}$ cells (63\% and $42 \%$, respectively) [23].

\section{Microinjection}

Microinjection refers to the use of a glass micropipette at a microscopic level to inject foreign molecules directly into living cells. Microinjection, as a simple mechanical process, has become a common laboratory method to deliver exogenous protein or DNA into single cells. To inject the CRISPR-Cas9 system directly into embryonic cells or other cells with a high duplicability and specificity microinjection was utilized [24, 25]

Microinjection has been employed to evaluate the geneediting efficacy of various plasmid-based CRISPR/Cas9 systems targeting the same gene due to its simplicity and accuracy [26].

There are numerous weaknesses of microinjection although these findings revealed that microinjection is an effective physical method for delivering plasmid-based CRISPR-Cas9 or Cas9 mRNA/sgRNA. First, as microinjection produces cell damage it needs a high level of sophistication and manual skills. Second, in each injection only a single cell can be targeted. Finally, this method is merely appropriate for a restricted number of cells [26].

2. Induced transduction by osmocytosis and propanebetaine (iTOP)

A new technique to deliver native proteins and other compounds into cells is iTOP.

Transduction of iTOP is beneficial for separate intracellular delivery of the Cas9 protein and sgRNAs, or direct delivery of RNPs. RNPs have been effectively delivered into different kinds of primary cells using iTOP.

In comparison to other approaches, including electroporation, cationic lipids and CPPs, a lower gene-editing effectiveness is produced by iTOP in main cells [26-28]. Furthermore, as the Cas 9 protein is merely soluble in high salt concentrations deployed in iTOP, it is not appropriate for in vivo applications.

\section{Mechanical cell deformation}

5. Hydrodynamic injection

6. Summary of numerous delivery systems for CRISPR-Cas9.

As previously discussed, several physical and non-viral delivery methods, such as electroporation, microinjection and lipid nanoparticles have been effectively used for the delivery of CRISPR-Cas9 systems. [12]

In 2016, the first clinical trial employing CRISPR-Cas9 indicated high promise of using CRISPR-Cas9 for treating 
diseases with genetic disorders. But, the CRISPR-Cas9 system may encounter the same difficulties associated with gene therapy and other nucleic acid-based therapeutics. Specially, three main obstacles in utilizing CRISPR-Cas9 for therapeutic applications in humans include ethical issues, off-target effects, lack of safe and effective delivery systems [12].

\section{DNA editing in brain disorder}

Diseases of central and peripheral nervous systems are referred to as neurological disorders. The most familiar disorders in the brain are autism, epilepsy, schizophrenia, dementia-related diseases such as Alzheimer and Parkinson, and brain tumor [29].

Brain tumors have been known as one of cancers with the highest death rate worldwide, although they occur less frequently (approximately 250,000 cases per year). One of the well-analyzed genetic diseases in the brain is Autism spectrum disorder (ASD) within the SFARI gene database [30], is Autism spectrum disorder (ASD). So far, 845 genes have been defined as an ASD-associated gene. Some natural CRISPR nucleases have been adjusted for mammalian genome editing [31]. These enzymes vary in the protein size, PAM requirement and induced DSB sites in the protospacer upon targeting to the genome. So far, three CRISPR nucleases that derive from Streptococcus pyogenes (SpCas9), Staphylococcus aureus (SaCas9) and Cpf1 (CRISPR from PrevotellaandFrancisella 1) have been utilized in genome editing in the mouse brain.

\section{Delivery into the brain}

The delivery of the Casgene and sgRNA is one key process of CRISPR-based genome editing in the brain. Various points should be considered for design of experiments. First, an appropriate expression system for sgRNA and Casneeds to be adopted. The expression systems that could be used are as follows: 1) plasmid expression vectors; 2) viral transduction; 3) in vitro transcribed CasmRNA and sgRNA; 4) riboprotein (RNP) complex comprising the recombinant Cas enzyme and sgRNA.

In vitro transcribed CasmRNA and sgRNA are frequently utilized for pronuclear microinjection of embryo. Especially, RNP approach facilitates a quick and ephemeral expression of Cas and sgRNA, thus potential effects of unintentional genomic integration are avoided. Second, electroporation and virus-mediated deliveries could be chosen, if appropriate for experimental purposes. The cargo size of virus packaging should be regarded in the virus delivery system. To control the size limitation of Adeno associated virus (AAV) packaging, SaCas9, a shorter version of Cas9, was utilized in the brain [32].

Furthermore, each method enables gene transduction into various cell types at separate developmental stages. For example, in/exo utero electroporation could be a gene transfection method appropriate for implementing in vivo mutagenesis in the developing embryonic brain, since it enables gene transduction into neural stem cells (NSCs) situated in the neuroepithelium [33-35]. One of well-studied human diseases caused by inherited (germline) and non-inherited (somatic) mutations is brain tumor because of frequent availability of clinical samples.

An RNA-guided gene targeting system on specific genomic loci is CRISPR/Cas9 which can bedesigned for applications beyond the nuclease activity of Cas9 [29].

In primary mouse neurons and freely behaving mice by fusion of another genome editing tool TALEN to different transcriptional regulators and functional domains of chromat in regulators, a light inducible control of endogenous gene expression was attained [36]. To infer novel function of mutated proteins in brain tumor format ion, CRISPRmediated insertion of a DNA donor fragment carrying diseaseassociated point mutations would be appropriate. Double strand DNAs with two homology arms on both termini have been traditionally used as a donor DNA for genome editing. However, homologous recombination-mediated donor DNA insertion happens at much less rate than NHEJ. Rather, NHEJ-mediated somatic LOF mutations could happen in targeted loci. Therefore, somatic gene transfer-based mouse modeling needs higher effectiveness of homology-directed repair, resulting in higher penetrance of tumor format ion assays. Current CRISPR-mediated genome editing studies also raisedappealing examples to produce tumorassociated genomic rearrangements. These genomic arrangements have been achieved with two or more various sgRNAs in vitro [37, 38].

Lately, in vivo delivery of Cas 9 and sgRNA by an adenovirus or lentivirus allowed induction of the Eml4-Alk inversion in chromosome 17 that has been reported occasionally in nonsmall cell lung cancers [39, 40].

Additionally, CRISPR-mediated in vivo deletion of a large genomic fragment has been reported as well [41], inspiring us to improve brain tumor models driven by genomic rearrangements. Although these improved CRISPR technologies would be appropriate for modeling of other genetic disorders, in vivo application of CRISPR might not be highly effective for induction of anticipated mutations. Still, low effectiveness of mutagenesis might be less disadvantageous in cancer research in comparison to studies on neurological disorders

To put it differently, oncogenic genetic alterations should be enhanced in advanced tumors. Thus, some insights into enquiries as to whether experimentally tested mutations are oncogenic will be provided by genomic analysis of developed tumors.

Moreover, one study indicated the attempt in development of the CRISPR library comprising 12 sgRNAs targeting per protein coding region [41].

Utilizing this high-ended library on 6 different human tumor cell lines shown core and context -dependent fitness genes. With suitable design of screening readouts, this novel library could be appropriate to seek innovative therapeutic approaches of neurodevelopmental disorders as well as brain tumors.

\section{DNA editing in liver}

Fibrolamellar hepatocellular carcinoma (FL-HCC) is a main liver cancer that mostly affects children and young adults with no concealed liver disease. A somatic, $400 \mathrm{~Kb}$ deletion on chromosome 19 that fuses part of the DnaJ heat shock protein family (Hsp40) member B1 gene (DNAJB1) to 
the protein kinase cAMP activated catalytic subunit alpha gene (PRKACA) has been constantly recognized in patients with FL-HCC. Nevertheless, the DNAJB1-PRKACA gene fusion has not been revealed to induce liver tumorigenesis. We utilized the CRISPR/Cas9 method to delete the syntenic region on chromosome 8 in mice to generate a Dnajb1-Prkaca fusion and monitored the mice for liver tumor development.

We provided CRISPR/Cas9 vectors designed for juxtaposing exon 1 of Dnajb1 with exon 2 of Prkaca to produce the Dnajb1-Prkaca gene fusion related to FL-HCC, or control Cas9 vector, through hydrodynamic tail vein injection to livers of 8-week-old female FVB/N mice. These mice did not have any other engineered genetic alterations and were not exposed to liver toxins or carcinogens.

Employing CRISPR/Cas9 technology, we found production of the Dnajb1-Prkaca fusion gene in wild-type mice to be adequate to begin formation of tumors that have many properties of human FL-HCC. Approaches as therapeutics for this form of liver cancer might be developed to block DNAJB1PRKACA [42].

\section{Crisper cas9 in breast cancer}

The CRISPR/Cas9 method has revolutionized the diagnosis and treatment of breast cancer. An invasive lobular breast carcinoma was taken as a model in which the CRISPR/ Cas9-mediated somatic genome editing tool confirms putative cancer drivers in vivo for the diagnosis [43].

For the diagnosis, we take an invasive lobular breast carcinoma was taken as a model in which the CRISPR/Cas9mediated somatic genome editing tool confirms putative cancer drivers in vivo [44].

For the treatment, we discuss the inhibition of breast cancer cell proliferation via a dominant negative mutation created by the CRSPR/Cas9-mediated targeting of oncogene HER2 [15, 44] .

Modeling of invasive lobular carcinoma (ILC) is facilitated by the emergence of CRISPR/Cas9-mediated somatic genome editing to confirm putative cancer drivers in vivo [43].

The second mo.st prevalent kind of human breast cancer is invasive lobular carcinoma which is responsible for $8-14$ $\%$ of all breast cancer cases [45-47]. This type of human breast cancer is described as discohesive epithelial cells invading the neighboring tissue in single-file patterns co-occur with copious fibroblasts and collagen deposition [15].

Genome editing can be performed in a quick, exact and efficient manner with the invention of the CRISPR/Cas9 technology. The potential applications of the CRISPR/Cas9 technology in functional interrogation of cancer-causing genes and cancer therapy have been widely investigated.

In this study, the utilization of the CRISPR/ Cas9 system to directly target the oncogene HER2 was demonstrated

Directing Cas9 to exons of the HER2 gene inhibited cell growth in breast cancer cell lines that harbor amplification of the HER2 locus.

The inclusion of PARP inhibitors potentiated the inhibitory effect. Unpredictably, the level of HER2 protein expression was not significantly affected by CRISPR-induced mutations.
Alternatively, CRISPR targeting appeared to exert its influence via a dominant negative mutation. This HER2 mutant meddle in the MAPK/ERK axis of HER2 downstream signaling.

Our study offers an innovative mechanism underlying the anti-cancer effects of HER2-targeting by CRISPR/Cas9, which is different from the clinical drug Herceptin. Furthermore, it opens up the opportunity that half-completed CRISPR targeting of particular oncogenes could still have therapeutic advantage by production of dominant negative mutants [44].

DNA editing in blood disorder

The main type of hemoglobin in human adults is hemoglobin A which comprises two alpha and two beta chains.

Sickle cell disease (SCD) is an autosomal recessive genetic disorder resulting from a single nucleotide mutation (A to T), and the glutamate-to-valine substitution at the sixth residue of the $\beta$-globin gene ( $\mathrm{HBB})$ results in the polymerization of hemoglobin and creates a sickle shape of red blood cells. As one of the molecular disorders with clearly elucidated molecular basis, SCD is a hotspot in the field of gene therapy. A lot of effort have been made to correct the mutant HBB gene in human iPSCs employing ZFNs and TALENs [48, 49] .Innovative causal mutations of hematological diseases are found and molecular diagnoses are accomplished more frequently with the popularization of next generation sequencing [50,51]. The pathogenic mechanisms of an increasing number of diseases have been identified at the gene level. A strong basis for gene therapy in hematological disorders is provided by these novel developments. CRISPR/Cas9 has been examined to treat hematological disorders such as sickle cell disease, $\beta$-thalassemia anemia, hemophilia, leukemia, and Fanconi anemia , and the exciting obtained outcomes provide us with full confidence to eradicate hematological genetic disorders. The CRISPR/Cas9 system was employed to target this pathogenic missense mutation in SCD patient-derived iPSCs. Researchers mutated allele in these iPSCs with a donor DNA template, which were then separated into erythrocytes effectively corrected one.

Approximately $6 \%-10 \%$ of the differentiated cells displayed features of reticulocytes. Furthermore, $16-\mathrm{kD} \quad \beta$-globin expression was noted in these erythrocytes [52].

Likewise, iPSCs obtained from human SCD patient keratinocytes were correctly edited by means of CRISPR/ Cas9 [16]. Patient-derived CD34+ hematopoietic stem and progenitor cells (HSPCs) were another choice for gene therapy. Over 18\% gene modification in CD34+ cells in vitro could be induced by CRISPR/Cas9. SCD-derived bone marrow CD34+ cells recovered the ability to generate wild-type hemoglobin A after Cas9 mRNA and an integrase defective lentiviral vector (IDLV) carrying a gRNA and a correct $\beta$-globin gene template were delivered into these cells [53]. Quite recently, a team of researchers corrected patient-derived HSPCs employing Cas9 ribonucleoproteins (RNP) and a homologous donor included in an adeno-associated viral vector. These cells could be separated into erythrocytes and express hemoglobin A messenger RNA [54]. Another team of researchers took a step further to transplant ex vivo treated human HSPCs into immunocompromised mice and discovered that these 
cells retained SCD gene edits during 16 weeks [55]. These preclinical studies demonstrated that gene edited iPSCs and HSPCs maintained the ability to change into full-grown progeny, which characterizes a research trend towards CRISPR-based cell therapies for treating hematological genetic disorders. Leukemia ASXL1 is an epigenetic modifier and tumor suppressor in chronic myeloid leukemia (CML), and its mutations are related to poor clinical result $[56,57]$. A mutant CML cell line KBM5 harboring an ASXL1 homozygous nonsense mutation (c.2128G > T, p.G710X) presented a lack of ASXL1 protein expression. In the presence of a 140-nt ssODN comprising the wildtype G nucleotide, the CRISPR/ Cas9 system corrected this mutation. The corrected cells reexpressed functional ASXL1 protein, and showed reduced cell development and increased myeloid differentiation. Furthermore, mice xenografted with corrected KBM5 cells demonstrated significantly longer survival than those with uncorrected xenografts [58]. This is an uncommon example of using CRISPR/ Cas9 to correct an acquired mutation included in leukemia. Over the last few years, much effort has been devoted to discovering a broader range of cancer driver genes, even in a patient-specific manner. Targeting driver genes is expected to provide twice the result with half the effort in cancer therapy.

With its simplicity and multiplex ability, CRISPR/ Cas9 can be utilized to correct acquired driver mutations or target numerous genes at the same time, which holds promise for attaining personalized cancer therapy. On the other hand, hematological disorders such as paroxysmal nocturnal hemoglobinuria harbor acquired mutations as well and can be the next candidates for gene therapy utilizing CRISPR/Cas9 [59].

\section{DNA editing in prostate cancer}

One of the most malignant types of tumor in developed countries is prostate cancer and mortality rate associated with prostate cancer has recently increased. The major causative agents of prostate cancer have been identified to be androgens. Following binding to the androgen receptor (AR), androgens play important role in the carcinogenesis of prostate cancers. ARs serve a significant role throughout all stages of prostate cancer, and preventing their function may help to slow growth of prostate cancer.

The androgen androgen receptor signaling pathway plays a significant role throughout all stages of prostate cancer. Following binding to the AR in prostate epithelial cells, androgens translocate to the cell nucleus. Once in the nucleus, androgens bind to androgen response elements upstream of the target genes, which results in DNA transcription, develops the unusual proliferation of prostate epithelial cells and allows carcinogenesis [60].

During the androgen sensitive stage of prostate cancer, the blockade or elimination of androgens avoids the binding of androgens to the AR, which may impede the advance of prostate cancer. But, at 18 months, prostate cancer becomes androgen independent. Thus, the tumor development may not be hindered employing androgen suppressors. Nevertheless, various studies have revealed that the AR maintains a role in androgen independent prostate cancer through $\mathrm{AR}$
Table 1. Disease and Gen on CRISPR-Cas9 technology for cancer therapy

\begin{tabular}{|c|c|c|}
\hline Disease & Gen & Delivery \\
\hline Liver cancer & Pten, $p 53$ & $\begin{array}{l}\text { Hydrodynamic } \\
\text { injection to deliver a } \\
\text { CRISPR plasmid } \\
\text { DNA expressing } \\
\text { Cas9 and sgRNAs }\end{array}$ \\
\hline $\begin{array}{l}\text { Lung } \\
\text { adenocarcinoma }\end{array}$ & $\begin{array}{l}\text { CD1 and C57BL/6J } \\
\text { (B6), p53+/- or } \\
\text { p53-/-, KrasLSL- } \\
\text { G12D/+ }\end{array}$ & $\begin{array}{l}\text { Adenoviral, } \\
\text { lentiviral }\end{array}$ \\
\hline Liver cancer & $\mathrm{FVB} / \mathrm{NJ}$ mice & $\begin{array}{l}\text { Hydrodynamic } \\
\text { injection }\end{array}$ \\
\hline Pancreatic cancer & $\begin{array}{l}\text { Editing of multiple } \\
\text { gene sets in pancre- } \\
\text { atic cells of mice }\end{array}$ & $\begin{array}{l}\text { Transfection based } \\
\text { multiplexed delivery } \\
\text { into mice }\end{array}$ \\
\hline Burkitt lymphoma & Arf/-E $\mu \mathrm{Myc}$ & $\begin{array}{l}\text { Lentiviral and } \\
\text { retroviral }\end{array}$ \\
\hline Colon cancer & ApcMin/+ & $\begin{array}{l}\text { Plasmid } \\
\text { transfection }\end{array}$ \\
\hline $\begin{array}{l}\text { Acute myeloid } \\
\text { leukemia (AML) }\end{array}$ & $\begin{array}{l}\text { p53 null HSPC } \\
\text { C57B1/6 mice or } \\
\text { heterozygous Flt3- } \\
\text { ITD knock-in mice }\end{array}$ & $\begin{array}{l}\text { Plasmid } \\
\text { Transfection } \\
\text { Lentiviral }\end{array}$ \\
\hline Lung metastasis & $\begin{array}{l}\text { KrasG12D/+; } \\
\text { p53-/-; Dicer1+/- }\end{array}$ & Lentiviral \\
\hline Breast cancer & $\begin{array}{l}\text { The stem cell marker } \\
\text { Cripto- } 1 \text { was shown } \\
\text { to be as a breast } \\
\text { target }\end{array}$ & $\begin{array}{l}\text { Plasmid transfection } \\
\text { into JygMC (mouse } \\
\text { cell line) }\end{array}$ \\
\hline $\begin{array}{l}\text { Gliobastoma } \\
\text { Medulloblastoma }\end{array}$ & $\begin{array}{l}\text { Postnatal PEI-medi- } \\
\text { ated transfection and } \\
\text { in utero electropora- } \\
\text { tion into mice }\end{array}$ & $\begin{array}{l}\text { Deletion of TSGs } \\
\text { (Ptch1, Trp 53, Pten } \\
\text { and Nf1) }\end{array}$ \\
\hline
\end{tabular}

gene mutation and gene amplification, and co-acts with co modulating factors to stimulate the unusual activation of other signaling pathways [61-63]. So, the growth of prostate cancer may be prevented by inhibiting AR.

The clustered frequently interspaced short palindromic repeats associated protein (CRISPR/Cas) system has lately become an extensively utilized gene editing technology [61, 64]. In comparison to earlier zinc finger nuclease (ZFN) and transcription activator like effector nuclease (TALEN) technologies, the CRISPR/Cas system is more extensively utilized in basic and in specific clinical studies [65]. A number of researchers have started to apply the CRISPR/Cas system in studies examining inherited genetic diseases, and certain studies have reached pertinent results [66-70].

The AR sgRNA guided CRISPR/Cas system could interrupt the AR at particular spots and hinder the development of androgen sensi $\neg$ tive prostate cancer cells; more studies revealed that the decreased cell proliferation was because of cellular apoptosis. The findings of this study recommended that the CRISPR/Cas system may be a valuable therapeutic approach for the treatment of prostate cancer. 
The CRISPR/Cas9 system has newly become a controversial subject in the field of gene editing. In comparison to ZFN and TALEN technologies, the CRISPR/Cas9 system has benefits in effectiveness and repeatability [71][72]. The CRISPR/Cas9 system has been commonly utilized in studies examining different tumors, comprising cervical cancer, liver cancer, lymphoma and prostate cancer.

This study utilized the CRISPR/Cas9 system to edit the AR gene, resulting in a restricted knockout in androgen dependent LNCaP cells. Stable LNCaP cells comprising sgRNA and Cas9 were made. These cells indicated a firm basis for more studies. Although a statistically significant difference was not observed, the effect of AR gene editing by CRISPR/Cas9 was noted in the LNCaP cells [73].

\section{References}

1. Lee W, Lee JH, Jun S, Lee JH, Bang D. Selective targeting of KRAS oncogenic alleles by CRISPR/Cas9 inhibits proliferation of cancer cells. Sci Rep. 2018;8(1):11879.

2. Hoorelbeke D, Decrock E, Van Haver V, De Bock M, Leybaert L. Calcium, a pivotal player in photodynamic therapy?. Biochim Biophys Acta Mol Cell Res. 2018;1865(11 Pt B):1805-1814.

3. Wen WS, Yuan ZM, Ma SJ, Xu J, Yuan DT. CRISPR-Cas9 systems: versatile cancer modelling platforms and promising therapeutic strategies. Int J Cancer. 2016;138(6):1328-1336.

4. Zhen S, Li X. Application of CRISPR-Cas9 for Long Noncoding RNA Genes in Cancer Research. Hum Gene Ther. 2019;30(1):39 .

5. Montaño A, Forero-Castro M, Hernández-Rivas JM, García-Tuñón I, Benito R. Targeted genome editing in acute lymphoblastic leukemia: a review. BMC Biotechnol. 2018;18(1):45.

6. Cassandri M, Smirnov A, Novelli F, et al. Zinc-finger proteins in health and disease. Cell Death Discov. 2017;3:17071.

7. Maeder ML, Gersbach CA. Genome-editing Technologies for Gene and Cell Therapy. Mol Ther. 2016;24(3):430-446.

8. Fan HC, Chi CS, Lee YJ, Tsai JD, Lin SZ, Harn HJ. The Role of Gene Editing in Neurodegenerative Diseases. Cell Transplant. 2018;27(3):364-378.

9. Ul Ain Q, Chung JY, Kim YH. Current and future delivery systems for engineered nucleases: ZFN, TALEN and RGEN. J Control Release. 2015;205:120-127

10. Chang CY, Ting HC, Su HL, Jeng JR. Combining Induced Pluripotent Stem Cells and Genome Editing Technologies for Clinical Applications. Cell Transplant. 2018;27(3):379-392.

11. Lee S, Lee CY, Lee J, Seo HH, Hwang KC. Cut and paste the genome: Genome editing for research and therapy. Journal of Cellular Biotechnology. 2015;1(1): 95-106.

12. Liu C, Zhang L, Liu H, Cheng K. Delivery strategies of the CRISPR-Cas9 gene-editing system for therapeutic applications. J Control Release. 2017;266:17-26.

13. Tao W, Yang A, Deng Z, Sun Y. CRISPR/Cas9-Based Editing of Streptomyces for Discovery, Characterization, and Production of Natural Products. Front Microbiol. 2018;9:1660.

14. Bhattacharjee R, Purkayastha KD, Adapa D, Choudhury A. CRISPR/Cas9 genome editing system in the diagnosis and treatment of cancer. Journal of RNA and Genomics. 2017;13(1): 585-591.

15. Yang H, Jaeger M, Walker A, Wei D, Leiker K, Weitao T. Break Breast Cancer Addiction by CRISPR/Cas9 Genome Editing. J
Cancer. 2018;9(2):219-231

16. Jubair L, McMillan NAJ. The Therapeutic Potential of CRISPR/ Cas9 Systems in Oncogene-Addicted Cancer Types: Virally Driven Cancers as a Model System. Mol Ther Nucleic Acids. 2017;8:56-63.

17. Jia C, Huai C, Ding J, et al. New applications of CRISPR/Cas9 system on mutant DNA detection. Gene. 2018;641:55-62.

18. Cong L, Zhang F. Genome engineering using CRISPR-Cas9 system. Methods Mol Biol. 2015;1239:197-217.

19. Niu Y, Shen B, Cui Y, et al. Generation of gene-modified cynomolgus monkey via Cas9/RNA-mediated gene targeting in one-cell embryos. Cell. 2014;156(4):836-843.

20. Zuris JA, Thompson DB, Shu Y, et al. Cationic lipid-mediated delivery of proteins enables efficient protein-based genome editing in vitro and in vivo. Nat Biotechnol. 2015;33(1):73-80.

21. Qin W, Dion SL, Kutny PM, et al. Efficient CRISPR/Cas9Mediated Genome Editing in Mice by Zygote Electroporation of Nuclease. Genetics. 2015;200(2):423-430.

22. Gori JL, Hsu PD, Maeder ML, Shen S, Welstead GG, Bumcrot D. Delivery and Specificity of CRISPR-Cas9 Genome Editing Technologies for Human Gene Therapy. Hum Gene Ther. 2015;26(7):443-451.

23. Liang X, Potter J, Kumar S, et al. Rapid and highly efficient mammalian cell engineering via Cas9 protein transfection. J Biotechnol. 2015;208:44-53.

24. Chang N, Sun C, Gao L, et al. Genome editing with RNA-guided Cas9 nuclease in zebrafish embryos. Cell Res. 2013;23(4):465472.

25. Crispo M, Mulet AP, Tesson L, et al. Efficient Generation of Myostatin Knock-Out Sheep Using CRISPR/Cas9 Technology and Microinjection into Zygotes. PLoS One. 2015;10(8):e0136690.

26. Nakagawa $Y$, Sakuma T, Sakamoto T, Ohmuraya M, Nakagata $\mathrm{N}$, Yamamoto T. Production of knockout mice by DNA microinjection of various CRISPR/Cas9 vectors into freezethawed fertilized oocytes. BMC Biotechnol. 2015;15:33.

27. D'Astolfo DS, Pagliero RJ, Pras A, et al. Efficient intracellular delivery of native proteins. Cell. 2015;161(3):674-690.

28. Suresh B, Ramakrishna S, Kim H. Cell-Penetrating PeptideMediated Delivery of Cas9 Protein and Guide RNA for Genome Editing. Methods Mol Biol. 2017;1507:81-94.

29. Feng W, Liu HK, Kawauchi D. CRISPR-engineered genome editing for the next generation neurological disease modeling. Prog Neuropsychopharmacol Biol Psychiatry. 2018;81:459-467.

30. Abrahams BS, Arking DE, Campbell DB, et al. SFARI Gene 2.0: a community-driven knowledgebase for the autism spectrum disorders (ASDs). Mol Autism. 2013;4(1):36.

31. Komor AC, Badran AH, Liu DR. CRISPR-Based Technologies for the Manipulation of Eukaryotic Genomes [published correction appears in Cell. 2017 Apr 20;169(3):559]. Cell. 2017;168(1-2):2036.

32. Ran FA, Cong L, Yan WX, et al. In vivo genome editing using Staphylococcus aureus Cas9. Nature. 2015;520(7546):186-191.

33. Saba R, Nakatsuji N, Saito T. Mammalian BarH1 confers commissural neuron identity on dorsal cells in the spinal cord. J Neurosci. 2003;23(6):1987-1991.

34. Kawauchi D, Taniguchi H, Watanabe H, Saito T, Murakami F. Direct visualization of nucleogenesis by precerebellar neurons: involvement of ventricle-directed, radial fibre-associated migration. Development. 2006;133(6):1113-1123.

35. Kawauchi D, Saito T. Transcriptional cascade from Math1 to Mbh1 and Mbh2 is required for cerebellar granule cell 
differentiation. Developmental Biology. 2008;322(2):345-354

36. Konermann S, Brigham MD, Trevino A, et al. Optical control of mammalian endogenous transcription and epigenetic states. Nature. 2013;500(7463):472-476.

37. Choi PS, Meyerson M. Targeted genomic rearrangements using CRISPR/Cas technology. Nat Commun. 2014;5:3728.

38. Torres R, Martin MC, Garcia A, Cigudosa JC, Ramirez JC, Rodriguez-Perales S. Engineering human tumour-associated chromosomal translocations with the RNA-guided CRISPRCas9 system. Nat Commun. 2014;5:3964.

39. Blasco RB, Karaca E, Ambrogio C, et al. Simple and rapid in vivo generation of chromosomal rearrangements using CRISPR/ Cas9 technology. Cell Rep. 2014;9(4):1219-1227.

40. Maddalo D, Manchado E, Concepcion CP, et al. In vivo engineering of oncogenic chromosomal rearrangements with the CRISPR/Cas9 system [published correction appears in Nature. 2015 Aug 27;524(7566):502]. Nature. 2014;516(7531):423-427.

41. Han J, Zhang J, Chen L, et al. Efficient in vivo deletion of a large imprinted lncRNA by CRISPR/Cas9. RNA Biol. 2014;11(7):829835.

42. Engelholm LH, Riaz A, Serra D, et al. CRISPR/Cas9 Engineering of Adult Mouse Liver Demonstrates That the Dnajb1-Prkaca Gene Fusion Is Sufficient to Induce Tumors Resembling Fibrolamellar Hepatocellular Carcinoma. Gastroenterology. 2017;153(6):1662-1673.e10.

43. Annunziato S, Kas SM, Nethe M, et al. Modeling invasive lobular breast carcinoma by CRISPR/Cas9-mediated somatic genome editing of the mammary gland. Genes Dev. 2016;30(12):14701480 .

44. Wang H, Sun W. CRISPR-mediated targeting of HER2 inhibits cell proliferation through a dominant negative mutation. Cancer Lett. 2017;385:137-143.

45. Martinez V, Azzopardi JG. Invasive lobular carcinoma of the breast: incidence and variants. Histopathology. 1979;3(6):467488.

46. Borst MJ, Ingold JA. Metastatic patterns of invasive lobular versus invasive ductal carcinoma of the breast. Surgery. 1993;114(4):637-642.

47. Wong H, Lau S, Cheung P, et al. Lobular breast cancers lack the inverse relationship between ER/PR status and cell growth rate characteristic of ductal cancers in two independent patient cohorts: implications for tumor biology and adjuvant therapy. BMC Cancer. 2014;14:826.

48. Sebastiano V, Maeder ML, Angstman JF, et al. In situ genetic correction of the sickle cell anemia mutation in human induced pluripotent stem cells using engineered zinc finger nucleases. Stem Cells. 2011;29(11):1717-1726.

49. Zou J, Mali P, Huang X, Dowey SN, Cheng L. Site-specific gene correction of a point mutation in human iPS cells derived from an adult patient with sickle cell disease. Blood. 2011;118(17):45994608

50. Boeke JD, Church G, Hessel A, et al. GENOME ENGINEERING. The Genome Project-Write. Science. 2016;353(6295):126-127.

51. Simeoni I, Stephens JC, Hu F, et al. A high-throughput sequencing test for diagnosing inherited bleeding, thrombotic, and platelet disorders. Blood. 2016;127(23):2791-2803.

52. Huang X, Wang $\mathrm{Y}$, Yan $\mathrm{W}$, et al. Production of GeneCorrected Adult Beta Globin Protein in Human Erythrocytes Differentiated from Patient iPSCs After Genome Editing of the Sickle Point Mutation. Stem Cells. 2015;33(5):1470-1479.

53. Hoban MD, Lumaquin D, Kuo CY, et al. CRISPR/Cas9-
Mediated Correction of the Sickle Mutation in Human CD34+ cells. Mol Ther. 2016;24(9):1561-1569.

54. Dever DP, Bak RO, Reinisch A, et al. CRISPR/Cas9 $\beta$-globin gene targeting in human haematopoietic stem cells. Nature. 2016;539(7629):384-389.

55. DeWitt MA, Magis W, Bray NL, et al. Selection-free genome editing of the sickle mutation in human adult hematopoietic stem/progenitor cells. Sci Transl Med. 2016;8(360):360ra134.

56. Grossmann V, Kohlmann A, Zenger M, et al. A deepsequencing study of chronic myeloid leukemia patients in blast crisis (BC-CML) detects mutations in $76.9 \%$ of cases. Leukemia. 2011;25(3):557-560.

57. Makishima $\mathrm{H}$, Jankowska AM, McDevitt MA, et al. CBL, CBLB, TET2, ASXL1, and IDH1/2 mutations and additional chromosomal aberrations constitute molecular events in chronic myelogenous leukemia. Blood. 2011;117(21):e198-e206.

58. Valletta S, Dolatshad H, Bartenstein M, et al. ASXL1 mutation correction by CRISPR/Cas9 restores gene function in leukemia cells and increases survival in mouse xenografts. Oncotarget. 2015;6(42):44061-44071.

59. Shi $\mathrm{H}$, Jiang $\mathrm{M}$, Wang Z. Comprehensive update on applications of CRISPR/Cas9 for hematological diseases. Int J Clin Exp Med10(8): 11409-11423.

60. Feldman BJ, Feldman D. The development of androgenindependent prostate cancer. Nat Rev Cancer. 2001;1(1):34-45.

61. Cohen MB, Rokhlin OW. Mechanisms of prostate cancer cell survival after inhibition of AR expression. J Cell Biochem. 2009;106(3):363-371.

62. Yuan X, Balk SP. Mechanisms mediating androgen receptor reactivation after castration. Urol Oncol. 2009;27(1):36-41.

63. Saraon P, Jarvi K, Diamandis EP. Molecular alterations during progression of prostate cancer to androgen independence. Clin Chem. 2011;57(10):1366-1375.

64. Mali P, Yang L, Esvelt KM, et al. RNA-guided human genome engineering via Cas9. Science. 2013;339(6121):823-826.

65. Pu J, Frescas D, Zhang B, Feng J. Utilization of TALEN and CRISPR/Cas9 technologies for gene targeting and modification. Exp Biol Med (Maywood). 2015;240(8):1065-1070.

66. Seeger C, Sohn JA. Targeting Hepatitis B Virus With CRISPR/ Cas9. Mol Ther Nucleic Acids. 2014;3(12):e216.

67. Lu XJ, Qi X, Zheng DH, Ji LJ. Modeling cancer processes with CRISPR-Cas9. Trends Biotechnol. 2015;33(6):317-319.

68. Matano M, Date S, Shimokawa M, et al. Modeling colorectal cancer using CRISPR-Cas9-mediated engineering of human intestinal organoids. Nat Med. 2015;21(3):256-262.

69. Tang H, Shrager JB. CRISPR/Cas-mediated genome editing to treat EGFR-mutant lung cancer: a personalized molecular surgical therapy. EMBO Mol Med. 2016;8(2):83-85.

70. Wang G, Zhao N, Berkhout B, Das AT. CRISPR-Cas9 Can Inhibit HIV-1 Replication but NHEJ Repair Facilitates Virus Escape. Mol Ther. 2016;24(3):522-526.

71. Ding Q, Regan SN, Xia Y, Oostrom LA, Cowan CA, Musunuru $K$. Enhanced efficiency of human pluripotent stem cell genome editing through replacing TALENs with CRISPRs. Cell Stem Cell. 2013;12(4):393-394.

72. Pattanayak V, Guilinger JP, Liu DR. Determining the specificities of TALENs, Cas9, and other genome-editing enzymes. Methods Enzymol. 2014;546:47-78.

73. Wei C, Wang F, Liu W, et al. CRISPR/Cas9 targeting of the androgen receptor suppresses the growth of LNCaP human prostate cancer cells. Mol Med Rep. 2018;17(2):2901-2906. 